\title{
POLYMER-MEMBRANE pH ELECTRODES AS INTERNAL ELEMENTS FOR POTENTIOMETRIC GAS-SENSING SYSTEMS
}

\author{
W. N. OPDYCKE, S. J. PARKS and M. E. MEYERHOFF* \\ Department of Chemistry, University of Michigan, Ann Arbor, MI 48109 (U.S.A.)
}

(Received 2nd June 1983)

\section{SUMMARY}

The use of polymer-membrane pH electrodes as internal sensing elements for the fabrication of inexpensive ammonia and carbon dioxide gas sensing systems is reported. The pH-responsive polymer membranes are prepared by incorporating tridodecylamine as the neutral carrier in plasticized poly(vinyl chloride) membranes. Both static and continuous-flow gas-sensing arrangements are described. For miniature static gas sensors, the internal polymer $\mathrm{pH}$ electrode can be made with or without an internal reference solution. In the latter case, the polymeric membrane is coated directly onto a graphite substrate. The polymer $\mathrm{pH}$ electrode can also be prepared in tubular form and used in conjunction with a gas dialysis chamber for automated continuous-flow determinations of carbon dioxide and ammonia. Slopes, response times, and reproducibility of these new gas-sensing systems are evaluated using optimized internal electrolytes, flow rates, and gas-permeable membrane materials. When appropriate reagents and materials are used, the static sensors exhibit slopes in the range $48-62 \mathrm{mV} /$ decade with potentials reproducible to less than $\pm 1.5 \mathrm{mV}$ at gas concentrations greater than $10^{-3} \mathrm{M}$.

The use of potentiometric gas sensors for the direct measurement of dissolved gases $\left(\mathrm{NH}_{3}, \mathrm{CO}_{2}, \mathrm{NO}_{2}\right.$, etc.) or as final detectors for indirect bioanalytical methods involving enzymes and microbial cells has grown rapidly in recent years [1-3]. Commercial gas sensors commonly utilized in such applications have glass $\mathrm{pH}$ electrodes as internal elements [3]. Although these sensors often exhibit theoretically predicted response properties and Nernstian behavior [4], the commercial devices are rather large (tip diameter $12-15 \mathrm{~mm}$ ) and expensive. Moreover, efforts to miniaturize these probes have been hampered by the fragile nature of the glass electrode and the flat bottom geometry required for functional static gas sensor designs.

In view of the limitations imposed by the use of glass electrodes, several workers have prepared gas sensors with alternative internal pH-responsive transducers. Mascini and Cremisini [5] reported the use of thermo-molded antimony-type $\mathrm{pH}$ electrodes as inner elements while workers at General Electric have patented the concept of using electrodes based on palladium or iridium oxide for the fabrication of miniature $\mathrm{CO}_{2}$ sensors [6]. Unfortunately, metal/metal oxide $\mathrm{pH}$ transducers may be subject to interferences from the redox environment of the sample (e.g., oxygen content) and this could limit their utility in the design of stable gas sensing systems. 
Over the past few years, our research efforts have focused on replacing the traditional glass-membrane electrodes in gas sensors with polymermembrane ion-selective electrodes [7-11]. Thus far, polymer membranes responsive to ammonium and carbonate ions have been utilized, along with appropriate internal buffer reagents, to prepare small, inexpensive, and rugged gas sensors for $\mathrm{NH}_{3}$ and $\mathrm{CO}_{2}$ [7-9]. The polymer-membrane electrodes have also been fabricated in a novel tubular form and used in conjunction with a gas dialysis chamber and flowing internal electrolyte to develop attractive automated continuous-flow gas sensing arrangements $[11,12]$.

Recently, Schulthess et al. [13] introduced a new pH-responsive polymer membrane electrode based on the incorporation of tridodecylamine in a poly(vinyl chloride) (PVC) matrix. Although the use of this polymer $\mathrm{pH}$ membrane has already been suggested for the fabrication of inexpensive gas sensors [8], it is the purpose of this paper thoroughly to evaluate and document this approach. Miniature static as well as automated $\mathrm{CO}_{2}$ gas-sensing arrangements are described and evaluated. The feasibility of utilizing polymercoated graphite $\mathrm{pH}$ electrodes within the miniature static probes is also examined. It will be shown that when constructed with appropriate internal electrolytes, gas-permeable membranes, etc., the resulting gas-sensing devices have response properties that compare favorably with the costly commercial glass $\mathrm{pH}$ electrode-based probes.

\section{EXPERIMENTAL}

\section{Apparatus and materials}

All potentiometric measurements were made using a Corning model 12, a Fisher Accumet model 620, or an Altex SelectIon $2000 \mathrm{pH} / \mathrm{mV}$ meter, and were recorded on either a Heath/Schlumberger model SR-204 or a Houston Instruments Omniscribe strip-chart recorder. Potentials for the polymer-membrane $\mathrm{pH}$ electrodes were evaluated against a saturated calomel reference electrode (SCE).

A schematic diagram for the automated $\mathrm{CO}_{2}$-sensing system is shown in Fig. 1. The ammonia system was essentially the same, except that the segmented stream required debubbling prior to passage through the dialysis chamber. The dialysis block and mixing coils were heated to $30^{\circ} \mathrm{C}$ for $\mathrm{CO}_{2}$ determinations. Two dialysis chambers were used for these studies, one with a channel/volume ratio of $1: 1$, and the other with a volume ratio of $1: 4$. These chambers were fitted with a polytetrafluoroethylene (PTFE) membrane (W. L. Gore Inc.) for the $\mathrm{NH}_{3}$-sensing system and a silicone polycarbonate membrane (General Electric) for the $\mathrm{CO}_{2}$-sensing system.

\section{Reagents}

All chemicals used were of reagent grade. Standard solutions and buffers were prepared with distilled, deionized water. 


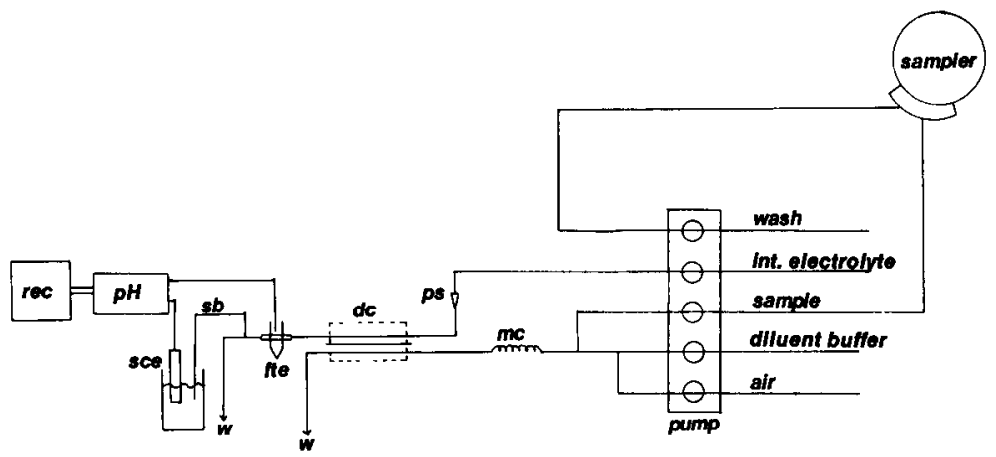

Fig. 1. Schematic diagram of automated carbon dioxide assay system: (rec) recorder; (pH) pH-mV meter; (SCE) saturated calomel reference electrode; (sb) salt bridge; (fte) flow-through electrode; (w) waste; (dc) dialysis chamber; (ps) pulse suppressor; (mc) 7 -turn mixing coil.

The pH-sensitive polymer membrane consisted of tridodecylamine, sodium tetraphenylborate (Eastman Kodak), poly(vinyl chloride) (chromatographic grade; Polysciences), and dibutyl sebacate (Sigma Chemical Co.).

In the automated system for carbon dioxide, the flowing internal electrolyte was a $0.001 \mathrm{M} \mathrm{NaHCO}_{3}$ solution prepared in $0.01 \mathrm{M} \mathrm{NaCl}$. The diluent stream was $0.1 \mathrm{M}$ citrate buffer ( $\mathrm{pH} 4.45$ ). Various concentrations of sodium hydrogencarbonate were prepared as standards for calibration purposes. The internal reference solution for the tubular electrode was $0.5 \mathrm{M}$ citrate buffer ( $\mathrm{pH} 4.45$ ) containing $0.1 \mathrm{M} \mathrm{NaCl}$.

For the automated ammonia-sensing system, the flowing internal electrolyte was $0.001 \mathrm{M} \mathrm{NH}_{4} \mathrm{Cl}$. The diluent was $0.001 \mathrm{M} \mathrm{NaOH}$. Several concentrations of ammonium chloride were prepared as standards for calibration purposes. The internal reference solution for the polymer $\mathrm{pH}$ electrode was $0.01 \mathrm{M} \mathrm{NH}_{4} \mathrm{Cl}$.

\section{Preparation of polymer $\mathrm{pH}$ electrodes and gas sensors}

Polymer $\mathrm{pH}$ electrodes were of two types, those with and those without internal reference solutions. Preparation of the polymer $\mathrm{pH}$ membrane and its incorporation into $\mathrm{pH}$ electrodes with internal reference solutions were described previously [8]. A schematic diagram of a pH electrode without the internal reference solution and the corresponding static gas sensor is shown in Fig. 2. This electrode consists of a $0.5-\mathrm{mm}$ diameter graphite rod (Pentel Hi-Polymer mechanical pencil lead) encased in a stainless steel syringe needle for strength and to provide electrical contact. This assembly is encased in heat-shrink tubing to provide insulation. The tip of the graphite rod is left exposed and is fitted with a piece of PVC tubing to provide a suitable surface for bonding the membrane. The $\mathrm{pH}$-sensitive membrane is applied dropwise (as a solution in tetrahydrofuran) to the tip. The gas sensors were constructed from pipet tips as described earlier [7]. The gas- 


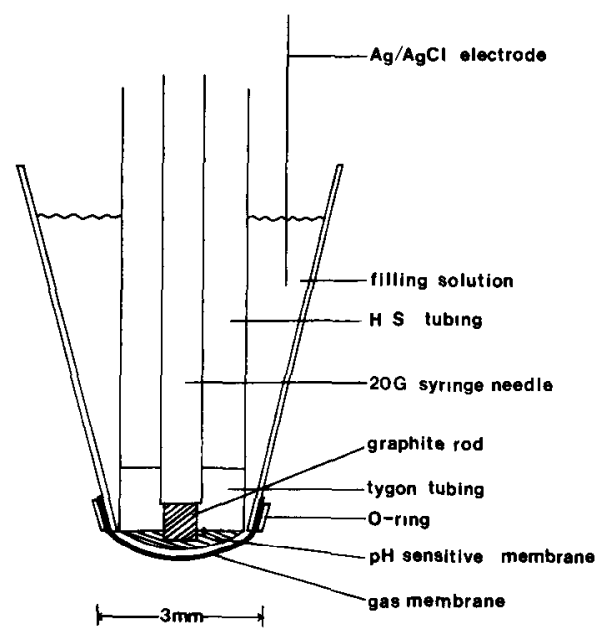

Fig. 2. Schematic diagram of static gas-sensing system utilizing a coated-graphite pH electrode.

permeable membrane used was microporous PTFE $(0.2-\mu \mathrm{m}$ pore; W. L. Gore and Assoc.). The tubular flow-through $\mathrm{pH}$ electrodes were prepared as described previously for ammonium and carbonate systems $[9,11]$.

These electrodes and corresponding gas sensors were evaluated for potentiometric response and analytical utility under the specific conditions described below.

Calibration curves for $p H$ electrodes and static sensors

Potentiometric response for the polymer-based $\mathrm{pH}$ electrodes was obtained in a solution which was $0.05 \mathrm{M}$ each in sodium acetate, tris-[hydroxymethyl]-aminomethane hydrochloride, and $N$-[2-acetamido]-2-aminomethanesulfonic acid. Microliter volumes of sodium hydroxide or hydrochloric acid were added to this solution and the actual $\mathrm{pH}$ was monitored after each addition with a calibrated glass pH electrode (Fisher 13-639-90). The potential of the polymer-membrane electrode (vs. SCE) was plotted vs. the measured $\mathrm{pH}$ value. Results for the static $\mathrm{CO}_{2}$ gas sensors were obtained from additions of sodium hydrogencarbonate to a $0.1 \mathrm{M}$ citrate buffer $(\mathrm{pH} 4.5)$ while data for the ammonia sensors were obtained from additions of ammonium chloride to $0.1 \mathrm{M} \mathrm{NaOH}$. Measurements were usually made at room temperature.

\section{RESULTS AND DISCUSSION}

Static $p H$ electrodes

Conventional polymer-based $\mathrm{pH}$ electrodes are those that have internal reference solutions. Their performance has been described $[4,13]$ and the response characteristics of our electrodes are essentially the same. There is, 
however, a possible problem when attempts are made to use conventional polymer $\mathrm{pH}$ electrodes (i.e., ones with internal reference solutions) in gassensing configurations. Analyte gas dissolved in the thin electrolyte film of the sensor may diffuse through the PVC ion-selective membrane and change the $\mathrm{pH}$ of the internal reference solution. This would result in errant or drifting response. Indeed, this behavior has been exploited to produce a hydrogen carbonate ion-selective electrode [14], which in reality functions as an inverted gas sensor. Thus, in order to produce useful gas sensors, the internal reference solution of the $\mathrm{pH}$ electrode must be buffered. Four buffers were evaluated to establish which would best maintain a constant $\mathrm{pH}$ in the internal reference solution. The buffers investigated were $0.1 \mathrm{M}$ and $1 \mathrm{M}$ phosphate $-\mathrm{HCl}, \mathrm{pH} 7.0$, and $0.1 \mathrm{M}$ and $1 \mathrm{M}$ citrate- $\mathrm{HCl}, \mathrm{pH} 4.2$. For this study, an Orion 95-02 gas-sensor body was used with a pH-sensitive polymer membrane as the gas-permeable membrane and the various buffers as the internal filling solutions. The $\mathrm{pH}$ of the thin buffer film was monitored with the internal glass $\mathrm{pH}$ electrode as the analyte gas $\left(\mathrm{NH}_{3}\right.$ or $\left.\mathrm{CO}_{2}\right)$ concentration was increased. Although all of the buffers worked well, the $1 \mathrm{M}$ citrate buffer yielded best results, holding the $\mathrm{pH}$ change to just 0.03 units even for gas concentrations as high as $10^{-2} \mathrm{M}$. Because it was found later that the $0.5 \mathrm{M}$ and $0.25 \mathrm{M}$ citrate buffers worked nearly as well, these were used for all subsequent experiments to prevent large osmotic effects.

Coated-substrate $\mathrm{pH}$ electrodes were also evaluated. Both graphite rods and platinum wires were used as substrates. For most experiments, graphite electrodes were used because it was easier to make them with the proper flat-bottom geometry desirable for good gas-sensor design and response. A calibration curve for the coated graphite $\mathrm{pH}$ electrode is shown in Fig. 3. The electrode responds logarithmically (typical slopes of $59-64 \mathrm{mV} / 10$-fold change in $\mathrm{H}^{+}$activity) to changes in hydrogen ion activity between $\mathrm{pH} 4$ and 10. Response was observed as low as $\mathrm{pH} 2$ and as high as $\mathrm{pH} 12$. Response times to reach equilibrium potentials were typically $15-20 \mathrm{~s}$ per unit $\mathrm{pH}$ change. Equilibrium was assumed when the potential drift was less than $0.4 \mathrm{mV} \mathrm{min} \mathrm{m}^{-1}$. Depending on the electrode, the reproducibility of potentials on consecutive calibrations can be as good as $\pm 1 \mathrm{mV}$ or as poor as $\pm 4 \mathrm{mV}$. Day-to-day reproducibility with regard to absolute potential values was found to be poor, although the slopes remained relatively constant. One possible explanation for this has to do with the nature of the inner reference potential of the coated substrate electrodes. This inner potential system is usually described as a metal/metal oxide half-cell with the necessary oxygen coming from diffusion of oxygen and water through the polymer membrane [15]. Changes in the oxygen concentration of the surrounding electrolyte will change the oxygen concentration in the membrane and perhaps alter the metal-oxide layer on the inner conductor. This will produce drifting or unstable potentials. Keeping the membrane hydrated apparently slows this process and helps to improve the reproducibility somewhat. In general, it was found that the coated-graphite $\mathrm{pH}$ electrodes were more stable than the coated-wire types. 


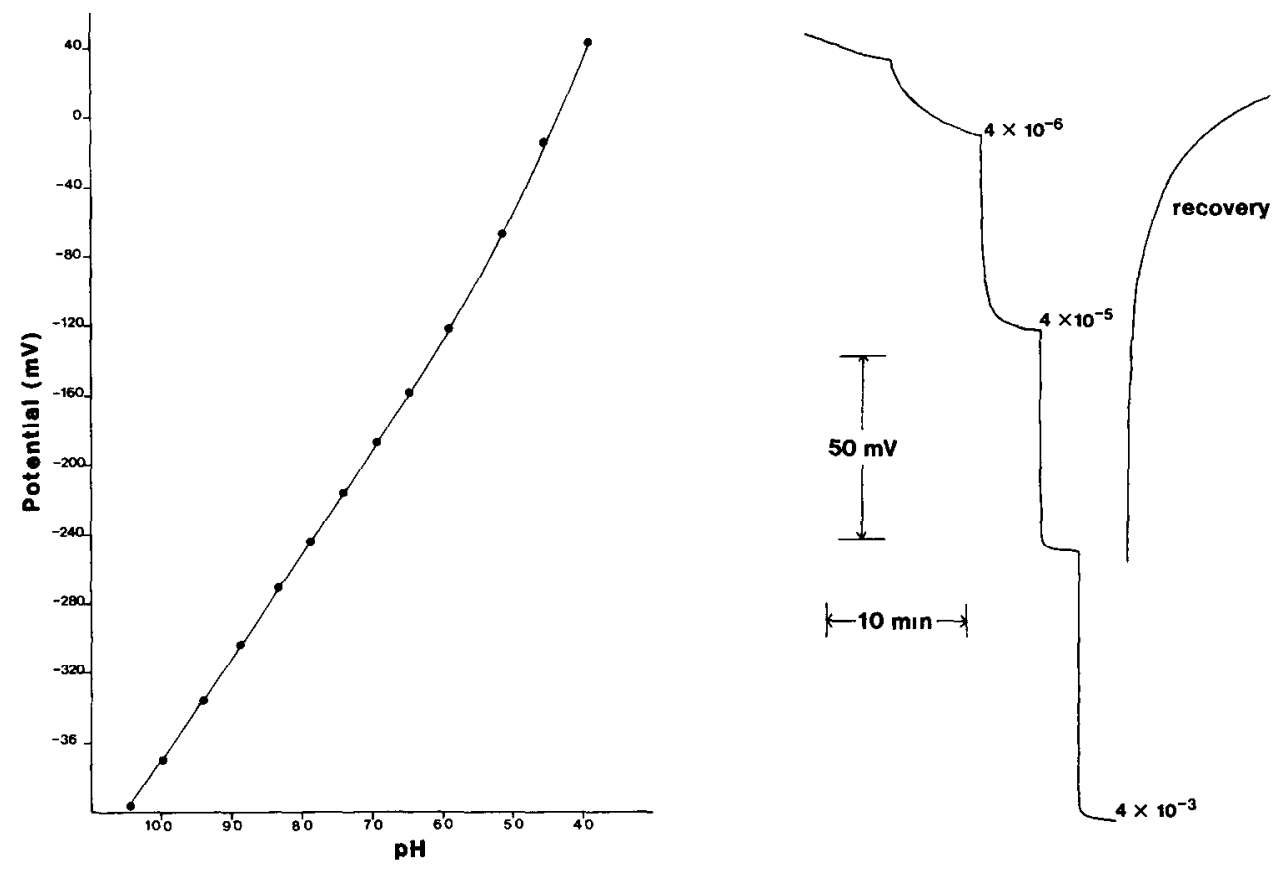

Fig. 3. Typical calibration curve for the coated-graphite $\mathrm{pH}$ electrode.

Fig. 4. Typical chart recording showing response and recovery times for the static ammonia sensor. Molar concentrations are given on the curves.

\section{Static gas sensors}

Gas sensors were constructed from both conventional polymer $\mathrm{pH}$ electrodes and coated-graphite electrodes. Both types of sensors displayed similar response properties. To avoid repetition, the response characteristics of the conventional polymer $\mathrm{pH}$ electrode-based sensors will be discussed in detail and only significant differences between the two will be mentioned.

An important parameter controlling gas-sensor response is the composition of the internal filling solution. The components of this solution and their concentrations determine the detection limits, slope, and time response of the sensor [4]. In order to obtain optimal response with the ammonia sensor, four internal filling solutions were evaluated. Two were commercial solutions (Orion 95-10-02 and $\mathrm{HNU}$ 10-10-01) and two were laboratory preparations $\left(0.01 \mathrm{M} \mathrm{NH}_{4} \mathrm{Cl}\right.$ in $0.1 \mathrm{M} \mathrm{NaCl}$ and $\left.0.1 \mathrm{M} \mathrm{NH}_{4} \mathrm{Cl}\right)$. The laboratory preparations performed better than the commercial ones, giving slopes that were typically $57-60 \mathrm{mV} /$ decade. The $0.1 \mathrm{M} \mathrm{NH}_{4} \mathrm{Cl}$ solution gave the best dynamic measurement range, but the solution which was $0.01 \mathrm{M} \mathrm{NH}_{4} \mathrm{Cl}$ in $0.1 \mathrm{M} \mathrm{NaCl}$ yielded lower detection limits and faster response times. The results described below were obtained using the latter solution.

The static ammonia gas sensor was evaluated for time response, linear response range, slope, and reproducibility. Figure 4 shows a typical recording 
for the $\mathrm{NH}_{3}$ sensor response as ammonium chloride was added to a $0.1 \mathrm{M}$ $\mathrm{NaOH}$ solution. The time response was slow at low concentrations $(5-10 \mathrm{~min})$ but improved rapidly with increasing gas concentration. It can also be seen that the recovery time of the sensor, when placed into a fresh solution of sodium hydroxide without ammonia, was similar to that of commercial ammonia sensors (i.e., $20 \mathrm{~min}$ ). A typical calibration curve for the ammonia gas sensor is shown in Fig. 5. Near-Nernstian response $(59-62 \mathrm{mV} /$ decade) was observed from $2 \times 10^{-5} \mathrm{M}$ to $4 \times 10^{-2} \mathrm{M}$ ammonia. The detection limit was estimated to be of the order of $1-2 \times 10^{-6} \mathrm{M}$. With regard to reproducibility, seven consecutive calibrations on a given day, yielded a slope of $61.2 \pm 0.8 \mathrm{mV}$ over a range from $4 \times 10^{-5} \mathrm{M}$ to $4 \times 10^{-2} \mathrm{M}$ ammonia. However, variations in the absolute potentials at equal analyte concentrations were somewhat greater, ranging from $\pm 2.5 \mathrm{mV}$ at a concentration of $4 \times$ $10^{-5} \mathrm{M}$ to $\pm 1.6 \mathrm{mV}$ at $4 \times 10^{-3} \mathrm{M}$. Day-to-day reproducibility of absolute potentials was poor, because of evaporation of the internal filling solution. For best results, the internal filling solution should be renewed daily, and as with any membrane electrode system, the sensor should be calibrated before and after measurements of unknowns.

The $\mathrm{CO}_{2}$ gas sensor was evaluated in a similar fashion. Again, four filling solutions were studied: HNU ISE 10-22-02, Orion 95-02-02, $0.01 \mathrm{M} \mathrm{NaHCO}_{3}$ in $0.1 \mathrm{M} \mathrm{NaCl}$, and $0.01 \mathrm{M} \mathrm{NaHCO}_{3}$ in $0.25 \mathrm{M} \mathrm{NaCl}$. The last solution was evaluated in an attempt to establish whether matching the osmolarity of the internal filling solution with that of the sample solution would reduce e.m.f. drift caused by diffusion of water vapor. Drift was reduced, but the performance of both of the laboratory-prepared solutions was inferior to that obtained with the commercial solutions. Operation with the commercial
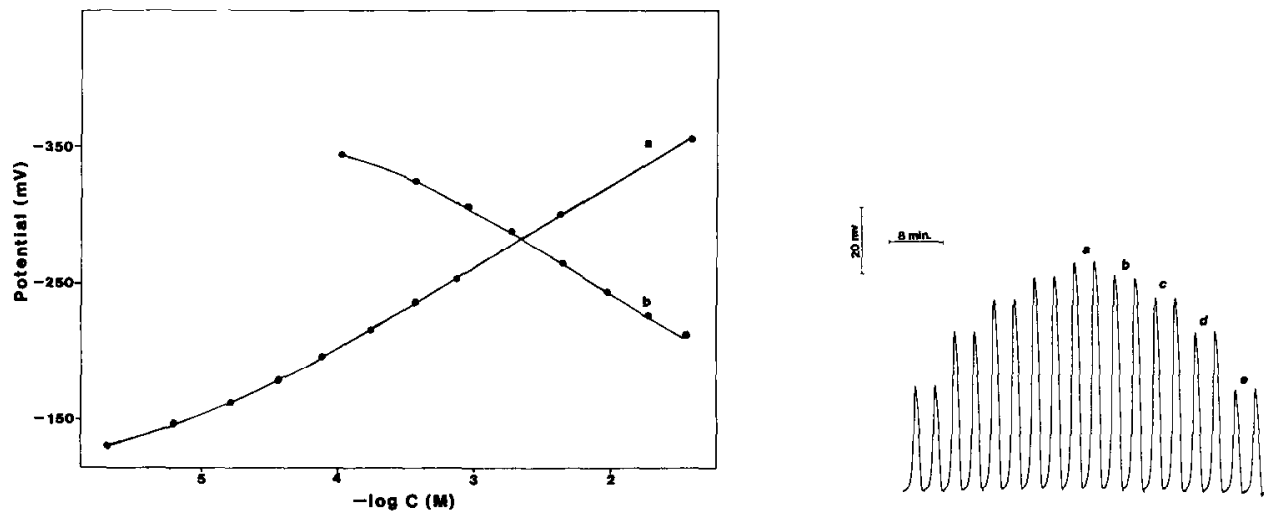

Fig. 5. Typical calibration curve for the static sensors based on an internal polymer membrane $\mathrm{pH}$ electrode : (a) ammonia; (b) carbon dioxide.

Fig. 6. Typical strip-chart recording obtained for automated $\mathrm{CO}_{2}$ system. Concentrations refer to $\mathrm{NaHCO}_{3}$ standards placed into the sampler: (a) $1 \times 10^{-3} \mathrm{M}$; (b) $8 \times 10^{-4} \mathrm{M}$; (c) $6 \times 10^{-4} \mathrm{M} ;$ (d) $4 \times 10^{-4} \mathrm{M} ;(\mathrm{e}) 2 \times 10^{-4} \mathrm{M}$. 
solutions yielded larger linear response ranges and improved slopes. The HNU internal filling solution was used for all subsequent experiments.

Another important parameter of the $\mathrm{CO}_{2}$ gas sensor is the selection of the gas-permeable membrane. Silicone rubber membranes are often used in commercial $\mathrm{CO}_{2}$ sensors rather than PTFE membranes. However, in the particular sensor configuration evaluated in this work, difficulties in attaching the silicone rubber membranes severely limited their usefulness; such membranes did not fit the shape of the sensor well and tended to leak. Thus, the PTFE membrane was used exclusively in the fabrication of the static gas sensors.

Response times for the static $\mathrm{CO}_{2}$ sensor ranged from $7 \mathrm{~min}$ at concentrations of $10^{-4}-10^{-3} \mathrm{M}$ to less than $1 \mathrm{~min}$ at concentrations greater than $10^{-3} \mathrm{M}$. Figure 5 shows a typical calibration curve for the polymer $\mathrm{pH}$ electrode-based $\mathrm{CO}_{2}$ sensor. Response is logarithmic (slopes of $48-53 \mathrm{mV}$ / decade) from $2 \times 10^{-4} \mathrm{M}$ to $4 \times 10^{-2} \mathrm{M} \mathrm{CO}_{2}$. Reproducibility is generally the same as for the ammonia gas sensor $( \pm 2.5 \mathrm{mV}$ at low gas concentrations, $\pm 1.5 \mathrm{mV}$ for concentrations greater than $10^{-3} \mathrm{M}$ ).

In general, the static sensors prepared with the polymer membrane $\mathrm{pH}$ electrodes possess response properties quite similar to those of conventional glass $\mathrm{pH}$ electrode-based probes. The one major difference is the larger variation in absolute potentials from day to day. However, provided that appropriate calibration procedures are used, the new devices appear to perform adequately.

\section{Automated systems}

The automated $\mathrm{NH}_{3}$ - and $\mathrm{CO}_{2}$-sensing systems described here utilize the same chemical detection principles as the static sensors described above. The configurations used to adapt the static sensors to the automated mode are variations of those described previously [11] based on a tubular ammonium ion-sensitive polymer membrane electrode as the detector. These variations along with response characteristics for each system are presented below.

Of the two systems constructed, the $\mathrm{CO}_{2}$ sensing system was the more extensively studied. In developing this system, the aim was to optimize the operating parameters to permit low-level $\mathrm{CO}_{2}$ measurements with good sample throughput. The continuous flow manifold shown in Fig. 1 differs in several ways from that described earlier [11]. A major change in the apparatus involved utilizing the sample stream without removing the air segmentation. A microporous teflon membrane was used in the gas dialysis chamber for the ammonia system [11]. Air can pass through that membrane resulting in large open circuit noise in the detection stream. Therefore, for the $\mathrm{CO}_{2}$ system, a homogeneous silicone polycarbonate membrane was used, through which air bubbles do not permeate. The advantage of using an airsegmented sample stream is that it allows for more of the sample $\mathrm{CO}_{2}$ to be detected. This is because any $\mathrm{CO}_{2}$ formed in the sample slug will equilibrate with the adjacent air bubbles. Removal of these air bubbles results in 
a substantial loss of the total $\mathrm{CO}_{2}$ sample. Using this arrangement, the signal resulting from low $\mathrm{CO}_{2}$ concentrations was greatly increased.

As Durst [16] has suggested, the sensitivity of the system can be enhanced by increasing the flow rate of the sample stream relative to the recipient stream. To this end, a flow rate ratio of $2: 1$ was used. This results in a concentration effect of the sample in the recipient electrolyte stream because this stream is flowing much more slowly than the sample stream. In an effort to improve further the sensitivity of the system, a special dialysis chamber was prepared with the recipient stream being $1 / 4$ the volume of the sample stream. If the system was allowed to reach equilibrium using this chamber, with solutions of equal $\mathrm{pH}$ in both streams, the concentration of sample in the recipient stream would be $80 \%$ of that originally present in the sample stream relative to only $50 \%$ for the usual channel ratio of $1: 1$. Therefore, although equilibrium is never actually achieved and the two streams do not actually have the same $\mathrm{pH}$ value, in practice, using the $1: 4$ chamber enables the recipient stream to contain a higher concentration of sample and thus results in a larger signal.

The concentration of the electrolyte in the recipient stream also plays an important role in the sensitivity of the system. Bailey and Riley [17] reported that the concentration of the electrolyte should be ten times higher than the lowest sample concentration to be quantified. This is because low levels of $\mathrm{CO}_{2}$ cannot produce a large enough $\mathrm{pH}$ change to be detected when the electrolyte is too concentrated. Several concentrations of electrolytes were examined and $10^{-3} \mathrm{M} \mathrm{NaHCO}_{3}$ was found to be the best for samples containing between $10^{-4} \mathrm{M}$ and $10^{-3} \mathrm{M} \mathrm{CO}_{2}$. Figure 6 shows a typical stripchart recording for the $\mathrm{CO}_{2}$ detector based on the tubular polymer membrane $\mathrm{pH}$ electrode, operating at 20 samples per hour. It can be seen that the automated system displays excellent precision with peak potentials generally reproducible to $\pm 1 \mathrm{mV}$. Resulting calibration graphs typically had slopes of 53-58 mV/decade with a logarithmic range from $2 \times 10^{-4}$ to $10^{-3} \mathrm{M} \mathrm{CO}_{2}$. For determinations of carbon dioxide at levels above $10^{-3} \mathrm{M}$, higher concentrations of sodium hydrogencarbonate in the recipient stream are recommended.

The ammonia-sensing system was constructed similarly to that described earlier [11] except that the ammonium ion-selective membrane used in the tubular electrode was replaced with a $\mathrm{pH}$-sensitive one. Good results were obtained with this system, but it was not as sensitive as the one utilizing the ammonium ion-selective electrode. That system had lower detection limits because it utilized a "buffer trap" effect (i.e., the recipient stream is buffered to an appropriate $\mathrm{pH}$ so that $\mathrm{NH}_{3}$ is converted to $\mathrm{NH}_{4}^{+}$, thus preventing any loss of analyte by diffusion). Obviously this could not be done in gas-sensing systems based on $\mathrm{pH}$ detection. Although measuring $\mathrm{pH}$ changes does not provide a system optimized for the determination of low levels of ammonia, it does offer a convenient method of determining higher concentrations over a larger concentration range. 
In summary, several designs for static and automated ammonia and carbon dioxide gas sensors based on polymer $\mathrm{pH}$-sensitive membrane electrodes have been evaluated. In view of their low cost, simple design, and ease of miniaturization, these gas sensors offer an attractive alternative to gas-sensing devices based on conventional glass $\mathrm{pH}$ electrodes.

The authors acknowledge the support of the National Institute of Health (Grant GM 2882-01). In addition, S. J. Parks acknowledges support from the Environmental Protection Agency (Grant EPA-R-80806301).

\section{REFERENCES}

1 M. E. Meyerhoff and Y. M. Fraticelli, Anal. Chem., 54 (1982) 27R.

2 G. A. Rechnitz, Science, 214 (1981) 287.

$3 \mathrm{M}$. Riley, in A. K. Covington (Ed.), Ion-Selective Electrode Methodology, Vol. II, CRC Press, Boca Raton, 1979, Ch. 1.

4 M. Mascini and C. Cremisini, Anal. Chim. Acta, 97 (1978) 237.

5 M. Mascini and C. Cremisini, Anal. Chim. Acta, 92 (1978) 277.

6 R. A. Macur, O. H. LeBlanc and W. T. Grubb, U.S. Pat. No. 3,905, 889, September 16, 1975.

7 M. E. Meyerhoff, Anal. Chem., 52 (1980) 1532.

8 M. E. Meyerhoff, Y. M. Fraticelli, J. A. Greenberg, J. Rosen, S. J. Parks and W. N. Opdycke, Clin. Chem., 28 (1982) 1973.

9 J. A. Greenberg and M. E. Meyerhoff, Anal. Chim. Acta, 141 (1982) 57.

10 M. E. Meyerhoff and R. H. Robins, Anal. Chem., 52 (1980) 2383.

11 Y. M. Fraticelli and M. E. Meyerhoff, Anal. Chem., 53 (1981) 992.

12 Y. M. Fraticelli and M. E. Meyerhoff, Anal. Chem., 55 (1983) 359.

13 W. Schulthess, Y. Shijo, H. V. Pham, E. Pretsch, D. Ammann and W. Simon, Anal. Chim. Acta, 131 (1981) 111.

14 R. J. J. Funck, W. E. Morf, P. Schultess, D. Ammann and W. Simon, Anal. Chem., 54 (1982) 423.

15 J. G. Schindler, G. Stork, H. J. Struh, W. Schmid and K. D. Karaschinski, Fresenius Z. Anal. Chem., 295 (1979) 248.

16 R. A. Durst, Anal. Lett., 10 (1977) 961.

17 P. L. Bailey, M. Riley, Analyst (London), 100 (1975) 1948. 